\title{
IMPACTO DEL PROGRAMA JÓVENES EN ACCIÓN EN LA FORMACIÓN INICIAL DE EDUCADORES EN MATEMÁTICAS
}

\section{IMPACT OF THE YOUNG IN ACTION PROGRAM IN THE INITIAL TRAINING OF MATHEMATICS EDUCATORS}

\section{Luz Silvana Maldonado Carvajal ${ }^{1}$ \\ Carlos Sebastián Gómez Vergel ${ }^{2}$ Mawency Vergel Ortega ${ }^{3}$}

Universidad Francisco de Paula Santander

\section{RESUMEN}

La investigación tiene como objetivo evaluar el impacto del programa Jóvenes en Acción en la permanencia y rendimiento académico de los beneficiarios y no beneficiarios de la Licenciatura en Matemáticas de la Universidad Francisco de Paula Santander. Se sustenta

1 Luz Maldonado: Magister en Educación matemática. Filiación: Universidad Francisco De Paula Santander Cúcuta, Colombia. correo: luzmaldonado@ufps. edu.co https://orcid.org/0000-0002-7751-7038

2 Carlos Sebastian Gomez ${ }^{2}$ Ingeniería Electrónica (Estudiante). Filiación: Universidad de Los Andes Bogotá, Colombia correo: cs.gomezv@uniandes.edu.co https:// orcid.org/0000-0002-6176-3613

3 Mawency Vergel Ortega ${ }_{3}^{3}$ Postdoctora en Ciencias sociales, niñez y juventud, Doctora en educación. Filiación: Universidad Francisco De Paula Santander, Cúcuta, Colombia. Correo: mawencyvergel@ufps.edu. co https://orcid.org/0000-0001-8285-2968 investigaciones sobre la permanencia y el rendimiento académico. Metodológicamente es un estudio con enfoque cualitativo con apoyo del análisis cuantitativo correlacional. Los resultados demuestran un impacto positivo en los estudiantes beneficiarios del programa jóvenes en acción. Se concluye que el apoyo familiar, los procesos de selección a través de pruebas saber y las ayudas económicas contribuyen con la permanencia estudiantil y al rendimiento académico en los estudiantes.

PALABRAS CLAVE. Permanencia estudiantil, rendimiento académico, programa Jóvenes en Acción 


\section{ABSTRACT}

The research aims to evaluate the impact of the Youth in Action program on the permanence and academic performance of the beneficiaries and non-beneficiaries of the Bachelor's degree in Mathematics at the Universidad Francisco de Paula Santander. The research is based on the permanence and academic performance. Methodologically, it is a study with a qualitative approach supported by correlational quantitative analysis. The results show a positive impact on the students who are beneficiaries of the Youth in Action program. It concludes that family support, selection processes through tests and financial aid contribute to student permanence and academic performance.

KEYWORDS. Student permanence, academic performance, youth program in Action.

\section{INTRODUCCIÓN}

La investigación evalúa el impacto del programa jóvenes en acción en la permanencia y rendimiento académico de los beneficiarios y no beneficiarios del plan de estudios de la Licenciatura en Matemáticas de la Universidad Francisco de Paula Santander en el periodo de ejecución del programa, II semestre del año 2014 al I semestre del año 2018. El programa Jóvenes en Acción es un apoyo del gobierno nacional de prosperidad social que ayuda a los jóvenes en condición de pobreza y vulnerabilidad, con la entrega de transferencias monetarias condicionadas para que puedan continuar sus estudios técnicos, tecnológicos y profesionales. En la Universidad Francisco de Paula Santander se inició en el año 2014 y actualmente apoya a un gran número de estudiantes inscritos en el programa, por lo cual, el estudio relacionado con el impacto del programa Jóvenes en Acción en la formación inicial de educadores en matemáticas se sostiene en dos variables: Permanencia estudiantil y rendimiento académico. En tal sentido, el soporte teórico explicita estos dos objetos de conocimiento, donde se hace una breve descripción de sus dimensiones en esta investigación.

La permanencia en la universidad se acoge al planteamiento del diccionario delaRealAcademia Española (2001) donde se alude a este vocablo como duración firme, constancia, perseverancia estabilidad e inmutabilidad; permanencia asociado a un valor que debe tener el estudiante cuando ingresa a los estudios universitarios, que le lleva a tener perseverancia para culminar su programa académico. La permanencia, acorde a Fonseca \& García (2016), se asocia con la acción de finalizar un plan de estudios. Así mismo, Chiroleu (2013) y Di Gresia (2009) plantean que la permanencia, está referida, a tres factores: acceso a los estudios universitarios, igualdad de oportunidades y abandono o deserción por distintas razones.

\section{Frenteal acceso a los estudios universitarios,} Chiroleu (2013) alude que no necesariamente supone una mayor democratización del nivel educativo, pues la igualdad, a su vez supone desiguales posibilidades de éxito a partir de orígenes socioeconómicos y educativos dispares. La autora acota que es posible distinguir entre un acceso formal a la institución y un acceso real al conocimiento, sólo posible a partir de condiciones mínimas de la calidad de la educación que permitan desarrollar un proceso efectivo de aprendizaje. Por su parte, López \& Moncada (2012) aseveran que el acceso a la universidad da cuenta de la exclusión social a través de la desigualdad de oportunidades para acceder a la enseñanza, y centran la discusión en la diferencia, con base en los orígenes sociales, la heterogeneidad y la calidad de los centros universitarios, y las motivaciones o aspiraciones de los sujetos. Según estos autores; dos líneas de análisis se han posicionado para analizar el acceso a la universidad: la teoría de la 
reproducción de Bourdieu \& Passeron (1979) y la teoría de la eficacia escolar de Weber (1971).

En relación con la teoría de la reproducción de Bourdieu \& Passeron (1979) en la incorporación a la universidad, todavía existe la preponderancia del examen como elemento para medir lo intelectual en el estudiante (Abril, Vergel, Gómez, 2020). Esta modalidad de ingreso para las medir las prácticas intelectuales, se puede ver con mayor énfasis en el sistema francés donde existe un peso funcional del examen en el sistema de enseñanza. En consecuencia, la idea central es de autonomía relativa del sistema gracias a la cual la enseñanza sirve de manera específica e insustituible a las estructuras sociales (Vergel, Gómez, 2019)), por lo cual el examen constituye un elemento fundamental para el acceso a los estudios superiores en el sistema educativo francés y quien no apruebe, difícilmente puede ingresar a los estudios universitarios.

Un análisis sociológico de dicha modalidad de acceso, según los autores, lleva a constituirse, efectivamente, en problemas propiamente sociales y pedagógicos. Puesto que, las dificultades surgidas del crecimiento del número de estudiantes que no ingresan al sistema, llevan a una relación de comunicación cuya forma y rendimiento están en función de la adecuación entre los niveles de ingreso y los niveles de recepción socialmente condicionados. Así pues, es en la distancia entre las exigencias implícitas del sistema de enseñanza y la realidad de su público donde se ve la función conservadora de la pedagogía tradicional, cuyo énfasis está en las pruebas de acceso al sistema.

De esta forma, la interpretación empirista de las relaciones observadas en los centros universitarios, deja claro que, como dicen Bourdieu \& Passeron (1979), la apariencia de fidelidad a lo real de una universidad, que pareciera proyectarse a la sociedad, más bien se limita al objeto aparente que le es cónsono; es decir, a una población escolar definida independientemente de su relación con la población, pues lo importante para su ingreso, es pasar los distintos niveles de acceso. Para salvar la trampa tendida por el sistema escolar, al dar solamente cupo una población determinada en el sector universitario, se hace alarde de los cursos y carreras dirigidos a la comunidad. También, surge entre las expectativas que el sistema educativo, y en general cultural, suscita en términos de liberación, de autorrealización y de movilidad social, nuevas maneras de acceso a la universidad y del mismo sistema escolar, pero siempre surgen mecanismos de selección y de exclusión.

Otro aspecto importante en el marco de los procesos acceso tiene relación con la teoría de la eficacia escolar de Weber, (1971) que plantea la posibilidad de que la universidad contribuya a revertir la desigualdad social. Este investigador encontró una serie de factores que, en contra de lo previsto, no tenían relación con el alto rendimiento, como por ejemplo, situaciones familiares como que los estudiantes vinieran de familias pobres o alejados de los centros universitarios. Asimismo, algunos factores académicos como el tamaño del aula, el agrupamiento de los alumnos por capacidad y las instalaciones físicas no son indicadores de alto desempeño y acceso. Murillo (2003) plantea tres indicadores de eficacia que deben tener las universidades: el principio de equidad que debe favorecer el desarrollo de todos y cada uno de sus alumnos, la idea de valor añadido referido a los elementos del contexto en el que se desarrolla, para que un centro sea considerado eficaz es necesario tener en cuenta tanto el rendimiento de los alumnos como la situación socio-económica y cultural de sus familias; y al desarrollo integral del alumno. Para Murillo (2003), una escuela no es eficaz si sus alumnos obtienen buenos resultados en pruebas de Lengua o Matemáticas pero no se preocupa de su formación en valores, bienestar 
- satisfacción. Una escuela será eficaz si desarrolla toda la personalidad de cada uno de sus alumnos.

Consecuentemente, surge para esta investigación, la igualdad de oportunidades, siguiendo los planteamientos de García (2007, P.67) se pueden mencionar tres concepciones de igualdad de oportunidades: meritocracia, igualitaria y compensatoria. Con respecto a la meritocracia Herrera (2013) interpreta la meritocracia como un elemento que legitima la exclusión de quienes pierden el cupo y no ingresan a la educación superior porque no cumplen con los requisitos. Pues, la meritocracia implica distribuir la educación según el talento de los estudiantes.

De igual modo, se plantea desde los postulados de García (2007) una "igualdad de oportunidades donde el criterio de distribución es la universalidad de la ciudadanía que pide que todos sean tratados de la misma manera" (p.67). De la misma manera, Herrera (2013) acota que, la desigualdad merecida, es la justificación solapada en los concursos de méritos que distribuyen la pobreza entre un gran número de demandantes de la asistencia educativa y de otros bienes antiguamente considerados públicos, pero están llenos de exclusión. El aplicar indiscriminadamente el parámetro de meritocracia, para simplemente llenar de alguna manera las pocas oportunidades que el Estado brinda a la población como proveedor legítimo de los servicios públicos, podría someter a la sociedad a la aplicación absurda de una desigualdad merecida. El otro elemento de igualdad de oportunidades está referido a la compensatoria; que, para García (2007) constituye el criterio de distribución, es decir, que la misma constituye la dificultad para conseguir determinados resultados, por lo que hay que concentrarse en los individuos que están en desventaja y en riesgo de no lograr un nivel adecuado de educación. Este último principio, es el más difícil de alcanzar.

Por otra parte, en cuanto a la dimensión abandono o deserción es necesario pensar según Guzmán, Durán, \& Franco (2009) que una intervención adecuada sobre el fenómeno de abandono, requiere un seguimiento sistemático de la deserción estudiantil. De igual manera, es importante su estudio desde diferentes perspectivas, como la institución de educación superior, programa académico y principalmente el estudiante, ya que los análisis desagregados permiten identificar con mayor precisión y oportunidad los factores que serán decisivos para el abandono de estudios y sobre los cuales puede intervenirse, logrando, por consiguiente, pertinencia, impacto y eficiencia en la aplicación de recursos.

Del mismo modo, Guzmán, Durán, \& Franco (2009) refieren que el proceso de deserción se compara la probabilidad de abandonar los estudios en cada periodo y la posibilidad de desertar o graduarse no es constante a lo largo del tiempo. En este sentido, la deserción o abandono comprende cuatro posibles resultados de interés, esto es: suspender los estudios por un tiempo y luego regresar, desertar, graduarse o continuar estudiando. Cada uno de estos resultados son afectados por variables exógenas y aunque los valores de estas variables son constantes en el tiempo, el efecto que tienen en la decisión de abandono cambia. Entre estas variables se pueden mencionar: género, raza, discapacidad, localización de la vivienda, edad de entrada a la institución, institución y puntaje en los exámenes de Estado.

Al evaluar el rendimiento académico, se analizan en mayor o menor grado los factores que pueden influir en él, generalmente se consideran, entre otros, factores socioeconómicos, la amplitud de los programas de estudio, las metodologías de enseñanza utilizadas, la dificultad de emplear una enseñanza personalizada, los conceptos previos que tienen los alumnos, así como el 
nivel de pensamiento formal de los mismos. El concepto de rendimiento tiene su acepción en el Diccionario de la Real Academia Española (2001) donde, en su entrada, se consigue que el mismo se relaciona con el producto o utilidad que rinde o da alguien o algo; o la proporción entre el producto o el resultado obtenido y los medios utilizados. De tal modo, que siguiendo a Guzmán (2012) y García \& Torres (2015), se puede establecer que el rendimiento académico está constituido por el nivel de conocimientos demostrado en una disciplina académica comparado con la norma de edad y nivel académico.

García \& Torres (2015), plantea que el rendimiento del estudiante debe ser entendido a partir de sus procesos integrales de evaluación, pues, la simple medición de los rendimientos alcanzados por los alumnos no provee, por sí misma, todas las pautas necesarias para la acción destinada al mejoramiento de la calidad educativa. Por otra parte, entre los aspectos caracterizadores del rendimiento académico se puede conseguir, una variedad, de acuerdo con distintos autores. En este sentido, se extrae una síntesis de los aspectos que lo caracterizan a partir de las ideas propuestas por Edel (2003), Guzmán (2012) y Guzmán \& otros (2009) quienes coinciden que, en términos del rendimiento académico la cantidad de variables se incrementa; que si la evaluación escolar, las calificaciones del alumno y el factor intelectual. Coinciden que la inteligencia humana no es una realidad fácilmente identificable. Además, toman en cuenta como características del rendimiento los éxitos y fracasos académicos, modos de relacionarse con los demás, proyecciones de proyectos de vida, desarrollo de talentos, notas educativas, resultados de test cognitivos, entre otros. Igualmente, consideran otro tipo de variables, al margen de las calificaciones y el nivel de inteligencia de los estudiantes, que aparentemente inciden en el rendimiento académico y que valdría la pena mencionar, como la familia, el contexto social, la salud, entre otros.

Sin embargo, con el propósito de no experimentar un agobio epistemológico en esta investigación y ante la naturaleza multifactorial de este fenómeno de estudio, de manera intencional, fueron seleccionados tres dimensiones: la motivación, las habilidades y las calificaciones, las cuales, de acuerdo con la perspectiva de la autora de la presente investigación, encuentran una vinculación significativa con el rendimiento académico y que pueden ser analizados en educación superior con la intención de poder evaluar sus implicaciones en el rendimiento escolar.

Robbins y Coulter (2005) mencionan que la motivación se refiere a todo aquello que hace que la gente actúe o se comporte de determinadas maneras. Es decir, la motivación corresponde a una serie de impulsos o deseos, los cuales fomentan cierto comportamiento Por lo tanto, cuando se estudia la motivación se parte de la premisa de que incide notablemente en todas las acciones que las personas pueden realizar. Sin embargo, cuando se habla de motivación en los estudiantes universitarios, no solo se debe hacer referencia a los estudiantes, sino también al docente como forjador de dicha motivación. Por lo cual, los profesores deben conocer las condiciones en las cuales a los estudiantes puede motivárseles para tengan éxito en sus estudios, al mismo tiempo que se cumplen los objetivos de cada programa.

En este orden de ideas, cuando se habla de motivación, no puede dejarse de lado la Teoría de la Motivación Humana de Maslow (1991) quien plantea que existe una jerarquía de necesidades y factores que motivan a las personas. Describe cinco categorías de necesidades y se construyen considerando un orden jerárquico ascendente de acuerdo a su importancia para la supervivencia y la capacidad de motivación. De acuerdo a este modelo, a medida que el 
hombre satisface sus necesidades, surgen otras que cambian o modifican el comportamiento del mismo; considerando que solo cuando una necesidad está razonablemente satisfecha, se disparará una nueva necesidad.

Entonces, de acuerdo con Maslow (1991), las necesidades fisiológicas son de origen biológico y están orientadas hacia la supervivencia del hombre, se consideran necesidades básicas e incluyen aspectos como necesidad de respirar, de dormir, de comer, de refugio. Cuando las necesidades fisiológicas están en su gran parte satisfechas surgen las necesidades de seguridad, orientadas hacia la seguridad personal, el orden, la estabilidad y la protección. En el tercer escalón emergen las necesidades de amor, afecto, pertenencia y la afiliación a un cierto grupo social y están orientadas, a superar los sentimientos de soledad. Posteriormente aparecen las necesidades de estima, una inferior que incluye el respeto de los demás, la necesidad de estatus, reconocimiento, reputación, y dignidad; y otra superior, que determina la necesidad de respeto de sí mismo, incluyendo sentimientos como confianza, competencia, logro, maestría, independencia y libertad. Finalmente, surgen las necesidades de auto-realización que se hallan en la cima de la jerarquía.

En relación a las habilidades Valiente (2000) y Capilla (2016) mencionan que la estructura cognitiva, representada por las ideas o conceptos existentes funciona como anclaje para establecer la conexión significativa con nuevos conceptos y crear habilidades para el aprendizaje. Empero, este proceso no es unidireccional sino bidireccional dado que tanto los conceptos o ideas preexistentes como los nuevos que se integran evolucionan conformando una nueva estructura. Es decir, como acota Gallego (1997), cuando se concreta la conexión lógica y sustancial entre un concepto prexistente en la estructura cognitiva y una nueva información o concepto presentado, este segundo adquiere significado, lo que favorece que ambos evolucionen y se pueda crear una plataforma para el desarrollo de habilidades en cualquier tema o situación. Al proceso mencionado, es a lo que Ausubel, Novak \& Hanesian (2009) denominan principio de asimilación. Así, al retomar la noción de habilidad presente en la estructura cognitiva del alumno, la misma servirá para la comprensión de todos los objetos de conocimiento.

Capilla (2016) establece que se puede indicar tres momentos donde se hace mención a las distintas habilidades que deben manejar los estudiantes, para resolver problemas matemáticos. $E \quad n$ el primer momento de aprendizaje procesos cognitivos intervienen al observar, que implica dar una dirección intencional a la percepción, atender, identificar, buscar y encontrar datos, elementos u objetos, recordar, recuperar la información almacenada en la memoria a largo plazo y relacionar o establecer la conexión de una idea, concepto, hecho o situación con otro. En el segundo momento de construcción de saberes intervienen procesos cognitivos tales como ordenar; entendido como la acción de disponer de manera sistemática un conjunto de datos a partir de un atributo determinado. Implica subhabilidades tales como reunir, agrupar, listar y seriar. Otro aspecto está referido con la capacidad de comparar, que implica establecer la relación de semejanza y diferencia entre objetos, conceptos o hechos que ayudan a relacionar o establecer una mayor conexión entre la información del exterior y la que posee el sujeto aprehensor. En el tercer momento los procesos cognitivos que favorecen la salida de la información, que comprueban no solo la conexión sino también el almacenaje en la memoria a largo plazo, la recuperación de la información y la adquisición de sentido y significados, son los siguientes: analizar, lo cual implica destacar los elementos básicos de una unidad de información y contempla 
subhabilidades tales como comparar, destacar, distinguir y resaltar; aplicar, donde el aprendiz es capaz de utilizar los conceptos e ideas en situaciones reales y específicas y evaluar que consiste en valorar a partir de la comparación entre un producto, los objetivos y el proceso. Implica subhabilidades tales como examinar, criticar, estimar y juzgar.

Con respecto a las calificaciones de los estudiantes como indicadores de rendimiento académico, Cerda (2008), Poggioli (2005) y Cabrales (2008) expresan que, probablemente una de las variables más empleadas por los docentes para aproximarse al rendimiento académico son las calificaciones. En sus estudios, estos investigadores atribuyen la importancia del tema a dos razones principales: la primera porque es uno de los problemas sociales y académicos que ocupan a los responsables profesionales de la educación, padres y madres de alumnos; y a la ciudadanía, en general, es la consecución de un sistema educativo efectivo y eficaz que proporcione a los alumnos el marco idóneo donde desarrollar sus potencialidades y la segunda, porque el indicador del nivel educativo adquirido ha sido, sigue y probablemente seguirán siendo las calificaciones escolares. A su vez, éstas son reflejo de la evaluación donde el alumno ha de demostrar sus conocimientos sobre las distintas áreas que el sistema considera necesarias y suficientes para su desarrollo.

En tal sentido, Cabrales (2008) alude a la evaluación de los aprendizajes en la educación superior, y desarrolla ideas donde menciona que la pertinencia se da en la medida en que los programas universitarios se convierten en un puente entre los saberes socialmente construidos y las prácticas evaluativas del docente, "...creando un enriquecimiento mutuo que permite a través de los procedimientos evaluativos, entre otras cosas, poner en evidencia las necesidades y los problemas de la sociedad..." (p.144), puesto que una evaluación adaptada a los estudiantes y referida a la problemática social actual, puede ayudar al aprendiz en la aplicación de los conocimientos a la vida real para comprender y transformar los problemas sociales, lo cual puede influir en un buen rendimiento académico.

Al disertar sobre prácticas evaluativas, integradoras del rendimiento académico, en la educación superior es necesario hacer mención a lo relacionado con las técnicas e instrumentos utilizados por los profesores universitarios para su ejecución. Al respecto, se siguen las ideas de Díaz Barriga y Hernández (2002), Poggioli (2005), Pérez Gómez (1993), Alves y Acevedo (1999) quienes coinciden en que las prácticas evaluativas en la acción pedagógica debe orientarla el docente en función de integrar por una parte, a todos los actores que conforman el hecho pedagógico y, por otra parte, cumplir con las exigencias académicas y formales que permitan la revisión de los planes y el contexto general de la dinámica del aula.

\section{PROGRAMA JÓVENES EN ACCIÓN}

Jóvenes en Acción es un programa de transferencias monetarias condicionadas del Gobierno Nacional implementado por Prosperidad Social en 2013, que pretende fomentar la formación de capital humano en la población vulnerable. El programa tiene cuatro objetivos específicos enfocados a la población de ingresos bajos: en primer lugar, busca incentivar la demanda de programas de formación técnica, técnica profesional, tecnológica, y profesional universitaria; en segundo lugar, pretende incrementar la permanencia en el sistema escolar; en tercer lugar, busca aumentar el logro educativo; y, por último, pretende fortalecer competencias sociales y laborales complementarias al programa de formación.

Jóvenes en Acción está constituido por dos componentes: el de Formación y el de 
Habilidades para la Vida. El componente de formación consiste en todas las actividades académicas específicas al programa educativo en el que se encuentra vinculado el estudiante. Además, incluye las competencias cognitivas y habilidades adquiridas en los Centros de Formación de educación superior.

El componente de Habilidades para la Vida busca fortalecer competencias no cognitivas relacionadas con habilidades personales $y$ profesionales. Este componente se organiza en tres módulos: el virtual, orientado a la dimensión personal; el presencial, dirigido a la dimensión social y laboral, constituido por talleres, seminarios y conversatorios; y el vivencial, orientado a las habilidades profesionales, compuesto por prácticas y voluntariados. Cada módulo es implementado de acuerdo con las necesidades específicas y la capacidad organizacional de cada región. Además, enfatizan en elementos como: el autoconocimiento, la comunicación asertiva, la empatía, la proactividad, el trabajo en equipo, la gestión del tiempo y de los conflictos.

El proceso de vinculación a Jóvenes en Acción se constituye por dos etapas: el registro al programa y la inscripción al programa de formación específico. El primero consiste en dos partes: el pre-registro, que se realiza por medio de convocatorias en los municipios focalizados en las direcciones regionales de Prosperidad Social, en los centros de formación del SENA y en las Instituciones de Educación Superior con convenio. En este paso, el individuo proporciona sus datos de identificación y contacto, carga su documento al Sistema de Información de Jóvenes en Acción (SIJA) y responde un cuestionario; la segunda parte consiste en el registro el cual se da una vez Prosperidad Social verifica los datos de identificación en el SIJA y el cumplimiento de los criterios de focalización territorial y poblacional. En caso de cumplir con los requisitos, el individuo pasa a estado registrado y tiene un año para inscribirse a algún programa de formación. Así pues, el joven participante es aquel que se encuentra registrado y, está inscrito en un programa de educación superior.

De otra parte, el individuo beneficiario debe asistir a Talleres de Participación en los cuales conoce las generalidades del programa y los compromisos adquiridos al formar parte de Jóvenes en Acción. Además, se le informa acerca de la verificación de los compromisos, la liquidación y entrega de los incentivos, y los canales de comunicación disponibles en el programa. Igualmente, los beneficiarios deben asistir a Jornadas de Enrolamiento Financiero para incorporarse con alguna de las entidades bancarias con convenio y adquirir algún tipo de servicio financiero que posibilite la entrega de las transferencias monetarias.

\section{METODOLOGÍA}

El marco en el cual se inserta el presente estudio se sustenta bajo el enfoque cualitativo, el cual lleva al tipo de investigación cualitativo. En cuanto al diseño (Briones $(1990,17-20)$ se enmarca bajo un estudio de campo, método fenomenológico. La población estuvo constituida por los estudiantes inscritos en la Licenciatura en Matemáticas de la Universidad Francisco de Paula Santander, en el periodo de ejecución del programa Jóvenes en Acción, II semestre del año 2014 al I semestre del año 2018. De donde fueron seleccionados 19 estudiantes beneficiarios y 19 no beneficiarios del programa jóvenes en Acción a quienes se les aplicó una entrevista estructurada y se cotejó con las calificaciones de los semestres que cursaba la población estudiantil beneficiaria del programa jóvenes en acción.

\section{RESULTADOS}

Al analizar los resultados relacionados con respecto a la variable permanencia en los 
estudios universitarios, en la dimensión acceso a los estudios, se consigue que la diferencia entre hombres y mujeres que acceden a la universidad no representa una diferencia significativa, aunque ingresan más hombres que mujeres a la carrera la Licenciatura en Matemáticas de la Universidad Francisco de Paula Santander. Entonces, a juicio de la investigadora, se infiere que motivado a la dificultad que siempre ha tenido el estudio de las matemáticas, hace que se inscriban más estudiantes del sexo masculino que del femenino. Este aspecto tiene relación con las ideas de Rama (1987) quien plantea que el acceso de las mujeres a los estudios universitarios se corresponde con la propuesta de una educación común obligatoria y gratuita, que incluyera a todos los niveles sociales, sin distinción de sexo.

Rama (1987) refiere que en América Latina, el acceso a los estudios universitarios de la mujer, se caracteriza por dos aspectos centrales: la expansión en la educación elemental en el marco de una función socializadora dirigida a la homogeneización de la población alrededor de un conjunto básico de códigos y valores y el desarrollo de la enseñanza media y superior dentro de una orientación humanística y enciclopedista destinada a la formación de élites dirigentes. Estas características, sin embargo, no produjeron un igual desarrollo del sistema educativo en Latinoamérica sino que en cada país tuvo sus particularidades. Pero en todos los casos favoreció la movilidad social de los estratos medios.

Otro aspecto que se consiguió fue el relacionado con la edad de los estudiantes que participaron consiguiéndose que la media se ubica en 22 años. En este sentido, se puede decir que la edad de los estudiantes es acorde al rango que debe existir en los estudios superiores en Colombia.

Por otra parte el análisis de la repitencia de los sujetos muestrales, refleja una repitencia inferior a la media, es decir que los estudiantes que repiten alguna asignatura son menos que los que no repiten. De ahí que la repitencia constituye, para la investigadora, un problema de gran interés, en tanto que se ha convertido en una de las mayores dificultades que obstaculiza el desarrollo intelectual, personal y el desarrollo del aprendizaje óptimo del estudiante, limitando su participación durante su proceso de formación.

En este sentido, Tejada, Villabona \& Ruiz (2013) establecen que una de las características del fenómeno de la repitencia es que tiene implicaciones académicas, personales, sociales, institucionales. Estos investigadores consideran que las cinco causas principales por las cuales se presenta la repitencia de las asignaturas son: bajo rendimiento académico y poco tiempo de dedicación al estudio, dificultades propias de la asignatura, falta de motivación y pocas bases del bachillerato. Consideran que no son determinantes para la repitencia causas como expectativas iniciales alrededor de la asignatura y malas relaciones con los compañeros de curso, por lo cual se asocia la repitencia a factores netamente académicos y correspondientes a características individuales de cada estudiante.

En cuanto al lugar de residencia durante los estudios universitarios, de los sujetos encuestados, mayoritariamente se consiguió que habitan en casa de sus padres y madres de familia. Esto es importante, por cuanto cuentan con el apoyo y ayuda directa de los padres para la prosecución de los estudios y culminarlos en el tiempo requerido. Si ello es así, entonces ¿qué papel juega el programa Jóvenes en Acción? Pues una de las características del programa es ayudar a los estudiantes con mayor necesidad. Pues de igual manera, los estudiantes provienen y viven, mayoritariamente, en la ciudad de Cúcuta. Es muy probable que la condición de vivir con los padres y madres en sus estudios universitarios lleve a que el análisis relacionado con la distribución que hacen los estudiantes del 
programa Jóvenes en Acción de los recursos asignados, un alto porcentaje determinó que los recursos del programa no le han brindado ningún beneficio.

Por otra parte, ante la interrogante sobre por qué se inscribió en la licenciatura en matemáticas de la Universidad Francisco de Paula Santander, para capacitarse en ésta área de conocimiento, un porcentaje alto, superior a la media, manifestó que es la carrera que le gusta y desea estudiarla. Este resultado, puede estar asociado a la escasa repitencia de la carrera, pues cuando joven hace lo que le gusta, los problemas que surgen son menores que aquellos que están obligados en un estudio determinado. Aspecto que lleva a que los estudiantes consideran que esta carrera le permitirá en un futuro una mejor calidad de vida. En este sentido, Vergel, Parra \& Martínez (2012) refieren que la capacitación se convierte en la herramienta esencial para el alcance de metas futuras que permite fortalecer las competencias del estudiante, generando diversos espacios de aprendizaje individual y colectivo, dirigido a mejorar y optimizar las habilidades, actitudes, aptitudes y conocimientos.

Otro aspecto asociado al acceso a los estudios universitarios está relacionado con la forma de acceso, en la cual el porcentaje significativo alto es a través de la prueba ICFES primera opción y prueba ICFES segunda opción. Este aspecto, también, podría estar influyendo en la escasa repitencia, pues ingresan al programa los alumnos que obtienen los mayores puntajes en las pruebas ICFES.

Siguiendo el análisis de la variable permanencia estudiantil, se presenta la discusión de la dimensión relacionada con la igualdad de oportunidades. Al respecto, los sujetos muestrales, de manera significativa, aluden que en la universidad se toma en cuenta el rendimiento académico para accesar a los programas sociales. Se consigue, además, que existe un alto porcentaje de estudiantes que tienen acceso a los programas de bienestar estudiantil que desarrolla la universidad, pero se mantienen neutrales ante la interrogante relacionada con la condición socioeconómica como aspecto para acceder a alguno de los programas sociales de bienestar universitario, pero plantean que no todos los estudiantes tienen igualdad de oportunidades.

Otra dimensión que lleva al análisis sobre la permanencia estudiantil, se refiere a la deserción o abandono. Según esta investigación, la deserción por período es el número de estudiantes que se debieron matricular en el período consultado para no ser declarados como desertores y no lo hicieron. Entonces, se puede observar que la deserción más alta se ubica en el semestre $2014-1$ con $17,39 \%$ y la más baja en el semestre 2015 -2, con 5,66\%. Lo cual lleva a inferir que los índices de deserción no son altos, esto es probable, a juicio de la investigadora, por cuanto los estudiantes tienen el apoyo directo de los padres ya que habitan en sus hogares, también por cuanto fueron seleccionador por el ICFES para su ingreso a la universidad, por lo cual cursan la carrera que desean estudiar.

Sobre los aspectos referidos a la deserción, Cabrera, Bethencourt, Alvarez \& González (2006, p.173) mencionan que la deserción estudiantil designa una variedad de situaciones que se presentan en el proceso educativo de un estudiante universitario que tienen como factor común la detención o interrupción de los estudios antes de culminarlos. Esto sucede, debido a situaciones como: deserción involuntaria por causas administrativas, abandonar un programa para iniciar otro en la misma institución, abandonar un programa para iniciar otro en otra institución, renunciar a los estudios universitarios para iniciar una vida laboral o interrumpir los estudios con la intención de regresar a ellos en el futuro.

Es innegable que la deserción es un fenómeno tan complejo que debe ser abordado desde 
diferentes perspectivas que permitan explicar sus orígenes, para ello se han planteados diferentes modelos que abordan esta problemática. En tal sentido, Cabrera, Bethencourt, Alvarez \& González (2006) y Tinto (1989) muestran algunos de los modelos usados para explicar las circunstancias que dan origen a la deserción estudiantil: i) modelo de adaptación, bajo esta mirada la deserción surge debido a la dificultad de adaptación e integración del estudiante al ambiente social de la universidad; ii) modelo estructural, se propone que la deserción es producto de las contradicciones entre varios subsistemas que componen el sistema social en su totalidad; iii) modelo económico, plantea una mirada de la deserción desde la individualidad de cada estudiante, se abandona debido a la elección del estudiante de una forma alternativa de invertir tiempo, energía y recursos que podría ofrecer mayores beneficios a futuro, $y$ iv) modelo psicopedagógico, afirma que son variables psicológicas y educativas las que inciden en mayor medida en el éxito o fracaso de los estudiantes.

En otro orden de ideas, surge el análisis de los resultados de la variable rendimiento académico, que se inicia con la dimensión motivación. En tal sentido, con respecto a la premisa si los sujetos muestrales piensan que al graduarse en la universidad conseguirá empleo de inmediato, los mismos se mantuvieron con porcentaje significativo en la categoría neutral, y en cuanto a la confianza en el futuro de la carrera para el ejercicio profesional existe un porcentaje alto en estar de acuerdo.

Con respecto a la motivación para el aprendizaje Alonso (2001) deja algunas ideas para la reflexión tales como que este constructo le la fuerza al estudiante para aprender. Si el aprendizaje no tiene sentido para el estudiante, simplemente éste no aprenderá. En tal sentido, reporta tres tipos de motivación para aprender; intrínseca, extrínseca y motivación condicional.
La motivación intrínseca es la fuerza impulsora que está detrás del aprendizaje social diario, los adolescentes se sumergen en estilos de vida específicos y los profesionales gastan mucho tiempo en su trabajo. La motivación extrínseca, enfatizando la educación como un medio para lograr algún otro propósito, y la motivación condicional, enfatizando que el aprendizaje puede ser poco placentero, pero que es una oportunidad para conocer otra gente y para lograr tener diversión durante el aprendizaje y después de este.

Por otra parte, cuando se plantea que los estudiantes piensan que la entrega de incentivos del programa Jóvenes en Acción permite mantenerse en el Programa de Licenciatura en Matemáticas, los resultados reflejan porcentajes significativos en las categorías de acuerdo y totalmente de acuerdo. Además, de ello los estudiantes reflejan resultados significativos en cuanto a la importancia del incentivo entregado por el programa Jóvenes en Acción para mejorar su desempeño académico donde los porcentajes altos se ubican en las categorías de acuerdo y totalmente de acuerdo.

En este orden de ideas, cuando se habla incentivos para que los estudiantes puedan permanecer en sus estudios universitarios, no puede dejarse de lado la Teoría de la Motivación Humana de Maslow (1991) quien plantea que existe una jerarquía de necesidades y factores que motivan a las personas. De acuerdo a este modelo, a medida que el hombre satisface sus necesidades, surgen otras que cambian o modifican el comportamiento del mismo; considerando que solo cuando una necesidad está razonablemente satisfecha, se disparará una nueva necesidad.

Con relación a la interrogante que permite verificar en los sujetos muestrales si piensan que cambiará de estrato social al graduarse y trabajará en la profesión como licenciados en matemática, de manera significativa los 
estudiantes esperan mejorar sus condiciones de vida al graduarse. Por ello, muchos aspiran, una vez que se gradúen poder comprar vehículo, adquirir vivienda, tener un empleo estable y ayudar a su familia, en escasos porcentajes, desean continuar estudios. En tal sentido, Robbins y Coulter (2005) mencionan que el aspecto mencionado tiene que ver con la motivación hacia todo aquello que hace que la gente actúe o se comporte de determinadas maneras. Es decir, la motivación corresponde a una serie de impulsos o deseos, los cuales fomentan cierto comportamiento,que en este caso está relacionada con los deseos de superación de los estudiantes.

Al respecto, Maslow (1991) refiere sobre las necesidades de estima, como la necesidad de estatus, reconocimiento, reputación, y dignidad; y otra superior, que determina la necesidad de respeto de sí mismo, incluyendo sentimientos como confianza, competencia, logro, maestría, independencia y libertad. Finalmente, surgen las necesidades de auto-realización que se hallan en la cima de la jerarquía. Necesidades estas que siempre están en la mente de los estudiantes, que aspiran, una vez graduados, autorrealizarse como seres humanos.

De igual modo, al analizar la dimensión habilidades matemáticas se puede conseguir que los estudiantes presentan las competencias requeridas, al menos en lo que responden en la entrevista, pues en porcentajes altos mencionan que tiene la competencia de observación o conexión de un concepto con otro, un porcentaje menor menciona que comprende los contenidos de todas las asignaturas, que busca tener un buen rendimiento académico y que desea prepararse mejor para el desempeño profesional.

En relación a las habilidades Valiente (2000) y Capilla (2016) mencionan que la estructura cognitiva, representada por las ideas o conceptos existentes funciona como anclaje para establecer la conexión significativa con nuevos conceptos y crear habilidades para el aprendizaje. Empero, este proceso no es unidireccional sino bidireccional dado que tanto los conceptos o ideas preexistentes como los nuevos que se integran evolucionan conformando una nueva estructura. Es decir, como acota Gallego (1997), cuando se concreta la conexión lógica y sustancial entre un concepto prexistente en la estructura cognitiva y una nueva información o concepto presentado, este segundo adquiere significado, lo que favorece que ambos evolucionen y se pueda crear una plataforma para el desarrollo de habilidades en cualquier tema o situación. En tal sentido, García \& Bartolucci (2007), refieren que las aspiraciones educativas están relacionadas con el acceso a la universidad, pues estas aspiraciones, no surgen por sí mismas, sino constituyen el resultado de una valoración de lo que es pertinente y posible para un estudiante a partir de la condición específica en la que se percibe a sí mismo y a su situación social y económica. Estos resultados se apoyan en Cabrera, Bethencourt, Alvarez \& González (2006) quienes refieren que uno de los aspectos de la deserción está relacionado con lo económico, pues plantean una mirada a la deserción desde la individualidad de cada estudiante, se abandona debido a la elección del estudiante de una forma alternativa de invertir tiempo, energía y recursos que podría ofrecer mayores beneficios a futuro, lo cual en esta investigación es altamente significativo, pues la deserción se ubica en umbrales muy bajos.

De igual manera, los estudiantes están de acuerdo o totalmente de acuerdo en el manejo de la competencia que permite establecer relaciones de semejanza y diferencia entre objetos, conceptos o hechos asociados a la matemática es fundamental en esta profesión. Asimismo, resuelven ecuaciones, funciones o cualquier tema asociado a la matemática con el uso de definiciones y formulas, las cuales las ven como competencias básicas para el 
ejercicio profesional de la matemática. Asimismo piensan que las aptitudes o habilidades para la matemática le permiten sacar buenas calificaciones. Entonces, se está en presencia, en términos conclusivos, de lo que Ausubel, Novak \& Hanesian (2009) denominan principio de asimilación. Es decir, que los estudiantes pueden retomar la noción de habilidad presente en la estructura cognitiva y la misma servirá para la comprensión de los objetos de conocimiento matemático.

En cuanto a desarrollo de pensamiento en ciencias y matemática, Capilla (2016) establece que se puede indicar tres momentos donde se hace mención a las distintas habilidades que deben manejar los estudiantes, para resolver problemas matemáticos. En el primero algunos de los procesos cognitivos que intervienen son observar para recuperar la información almacenada en la memoria a largo plazo y relacionar o establecer la conexión de una idea, concepto, hecho o situación con otro. En el segundo momento de construcción de saberes intervienen procesos cognitivos tales como ordenar. Otro aspecto está referido con la capacidad de comparar, que implica establecer la relación de semejanza y diferencia entre objetos y en el tercer momento los procesos cognitivos que favorecen la salida de la información, que comprueban no solo la conexión sino también el almacenaje en la memoria a largo plazo, la recuperación de la información y la adquisición de sentido y significados.

En cuanto a la dimensión calificaciones del estudiante, se puede observar que los promedios son más altos en los beneficiarios del programa que en los no beneficiarios. Algunos semestres son más significativos que otros, por lo cual, se puede decir que si existe un impacto positivo del programa Jóvenes en Acción en la permanencia y rendimiento académico de los estudiantes.

\section{CONCLUSIONES}

Con relación a la variable permanencia estudiantil se ha conseguido que el acceso a los estudios en la universidad a través de las pruebas Saber tiene un efecto positivo para que el estudiante se mantenga en sus estudios universitarios.

El acceso se construye a partir de dimensiones, como el género, pues se siguen identificando diferencias entre las aspiraciones de hombres y mujeres, y la edad, puesto que tener 18 años o menos al término del bachillerato puede considerarse más adecuado para continuar estudiando; el apoyo familiar, dado que la interacción entre padres e hijos durante los estudios universitarios constituye un aliciente valioso para la permanencia en la universidad. En este sentido, se pone en juego las condiciones sociales, económicas, el origen familiar y el lugar de residencia.

En cuanto a la igualdad de oportunidades, se retoma, que el acceso a la universidad se desarrolla de manera meritocrática, los estudiantes cristalizan sus aspiraciones educativas en el tránsito e incorporación hacia la educación superior, de manera más significativa, si saben que su ingreso fue sus propios méritos, mostrando un reconocimiento y adaptación con el entorno escolar y social, aunque en este proceso se enfrentan a dificultades académicas, económicas o familiares que resuelven de alguna forma, con el fin de continuar sus estudios de educación superior y además muestran iniciativa para la búsqueda de autonomía al combinar el estudio y algún tipo de trabajo que les permita continuar estudiando, aunque este último no sea indispensable para sostener sus estudios. Estos hallazgos ponen a los estudiantes al ingresar a la institución en situación de aprender y comprender un nuevo escenario y su rol en él, en el que cobran significado sus aspiraciones educativas y expectativas y se convierten en parte del anclaje de su vida futura. 
El abandono o deserción no es altamente significativa en semestres primero a séptimo con variable influyente la situación familiar y económica de los estudiantes, quienes a su vez reciben ayuda financiera, tanto de la universidad, como del programa jóvenes en acción, así como de otros organismos, se podría inferir que estos aspectos juegan a favor de la permanencia de los alumnos en la universidad.

Con relación a la variable rendimiento académico, se observa una motivación intrínseca, la cual tiene un papel predominante en los estudiantes universitarios en lo que se refiere a su desempeño, prevaleciendo así los motivos relacionados con el estatus, la movilidad social y el deseo de superación, pues se puede apreciar una escasa repitencia en los sujetos muestrales. Resultado asociado a dimensiones sociales al convivir con sus padres y estudiar la carrera que les gusta.

El desempeño se ha relacionado con las aspiraciones educativas. El deseo de ingresar a la educación superior es más fuerte entre quienes obtienen mejores resultados. Existen además variables relacionadas con la familia que también han mostrado estar relacionadas con las aspiraciones de los jóvenes. Con respecto a la Dimensión calificaciones se puedo obtener que si existe diferencia significativa entre los promedios de los beneficiarios del programa y los que no lo son. En líneas generales se puede decir que el impacto del programa Jóvenes en Acción es altamente positivo en los estudiantes beneficiarios pues se observan elementos indicadores tales como, baja deserción y altas calificaciones, con un promedio significativamente alto con respecto a los no beneficiarios.

\section{REFERENCIAS BIBLIOGRÁFICAS}

Academia, Real Española. (2001). Diccionario de la Real Academia Española. Versión en línea. Disponible en: http://buscon.rae.es/diccionario/ drae.htm

Alonso, J. (2001). Motivar a los estudiantes y enseñar a motivarse. Madrid: Santillana.

Altamirano, A., Hernández, E. \& Soloaga, I. (2012). Aspiraciones educativas y entorno socioeconómico. Una aplicación para el caso de los jóvenes de la Ciudad de México. Recuperado de http://papers.ssrn.com/sol3/ papers.cfm?abstract_id=2386849

Alves, E. \& Acevedo, R. (1999). La evaluación cualitativa. Reflexión para la transformación de la realidad educativa. Valencia: Cerined.

Ausubel, D. Novak, J. \& Hanesian, H. (1983). Psicología educativa. Un punto de vista cognoscitivo. México. Editorial Trillas.

Bourdieu, P. \& Passeron, J.C. (1979). La Reproducción. Elementos para una teoría del sistema de enseñanza. Barcelona: Editorial Laia, S.A.

Briones, G. (2000). La investigación en el aula y en la escuela. Bogotá: convenio Andrés Bello.

Cabrales, O. (2008). Contexto de la evaluación de los aprendizajes en la educación superior en Colombia: Sugerencias y alternativas para su democratización. Revista Educación y Desarrollo Social. Volumen II - No. 1 enero junio.141-165. Bogotá. Documento en línea: http://www.postgradoune.edu.pe/

Cabrera, L., Bethencourt, J. T., Alvarez, P. P., \& González, A. M. (2006). El problema del abandono universitario. RELIEVE, 12(2), 171-203, Recuperado el 12 de marzo de 2013, de http://www.uv.es/relieve/v12n2/ relievev12n2_1eng.pdf 
Capilla, R. (2016). Habilidades cognitivas y aprendizaje significativo de la adición y sustracción de fracciones comunes. Cuadernos de Investigación Educativa, 7 (2), 49-62. Recuperado de: http://www.redalyc.org/ comocitar.oa?id=443649571004.

Cerda, H. (2008). El Proyecto de Aula. El aula como un sistema de investigación y construcción de conocimientos. Bogotá. Editorial Magisterio.

Chiroleu, A. (2013). Políticas públicas de Educación Superior en América Latina: ¿democratización o expansión de las oportunidades en el nivel superior?. Espacio Abierto, 22 (2), 279-304. Recuperado de: http:// www.redalyc.org/articulo.oa?id=12226914006

Di Gresia, Luciano (2009). Educación universitaria: acceso, elección de carrera y rendimiento. La Plata: Univ. Nacional de La Plata. Recuperado de: http://sedici.unlp.edu.ar/ handle/10915/34204.

Díaz Barriga, F. y Hernández, G. (2002). Estrategias docentes para un aprendizaje significativo. México: McGraw Hill.

Edel, R. (2003). El rendimiento académico: concepto, investigación y desarrollo REICE. Revista Iberoamericana sobre Calidad, Eficacia y Cambio en Educación, vol. 1, núm. 2, julio-diciembre, 2003. Red Iberoamericana de Investigación Sobre Cambio y Eficacia Escolar. Madrid, España. Recuperado de: http://www. redalyc.org/pdf/551/55110208.pdf.

Escobar, M. (2006). La investigación sobre juventud en Colombia: construcción de los sujetos desde los discursos especializados. Revista Actualidades Pedagógicas Vol. 2 No 1/Enero-junio. Recuperado de: file:///C:/Users/Add/ Downloads/1833-Texto\%20del\%20art\%C 3\%ADculo-3612-1-10-20130129.pdf.
Figuera, P. Dorio, I. y Forner, A. (2001). Las competencias académicas previas $y$ el apoyo familiar en la transición a la universidad. Revista de Investigación Educativa, 21(2), 349-369.

Fonseca G., \& García, F. (2016). Permanencia y abandono de estudios en estudiantes universitarios: un análisis desde la teoría organizacional. Revista de la Educación Superior. Volúmen 45. Recuperado de: www.redalyc.org/pdf/604/60447470003. pdf

Gallego Badillo, R. (1997). Saber Pedagógico. Santafé de Bogotá: Magisterio

García-Castro, G. \& Bartolucci, J. (2007). Aspiraciones educativas y logro académico: un estudio de caso sobre características y condiciones sociales de los estudiantes de la UAM. Revista Mexicana de Investigación Educativa, 12(35), 1267-1288.

Guzmán, C., \& otros (2009). Deserción estudiantil en la Educación Superior en Colombia. Metodología de seguimiento, diagnóstico y elementos para su prevención. Bogotá: MEN.

Guzmán, C., Durán, D., \& Franco, J. (2009). Deserción estudiantil en la educación superior colombiana. Metodología de seguimiento, diagnóstico y elementos para su prevención. Bogotá: Ministerio de Educación Nacional.

Guzmán, M. (2012). Modelos predictivos y explicativos del rendimiento académico universitario: caso de una institución privada en México. Universidad Complutense de Madrid. Facultad de Educación. Recuperado de: http://eprints. ucm.es/15335/1/T33748.pdf. 
Hernández y otros (2008). Metodología de la investigación. Mcgraw-Hill/ interamericana. México.

Herrera, J. (2013). Ética, Equidad Y Meritocracia en la Mercantilización de la Educación Superior Colombiana. Revista Latinoamericana Bioética. Volumen 13, Número 1 Edición 24, Páginas 8-17.

López, C. M. y Moncada, L. Z. (2012). Expectativas de acceso a la universidad en los jóvenes de sectores populares de Bogotá. Educ. Vol. 15, No. 3, 383-409. Bogotá: Universidad de la Sabana.

Maslow, A. (1991). Motivación y personalidad. Madrid: Ediciones Díaz de Santos

Murillo, J. (2003). El movimiento de investigación de Eficacia Escolar. En F.J. Murillo (Coord.). La investigación sobre Eficacia Escolar en Iberoamérica. Revisión internacional del estado del arte. Bogotá: Convenio Andrés Bello.

Novak, J \& Hanesian, H. (2009). Hacia una teoría de la educación. Buenos Aires: Kapelusz.

Orjuela Abril, J. L., Vergel Ortega, M., \& Gómez Vergel, C. S. (2020). Desarrollo de competencias de innovación en estudiantes de cálculo a traves de proyectos de creación en tiempos de asesoria en casa. Revista Boletín Redipe, 9(5), 77-87. https://doi. org/10.36260/rbr.v9i5.976

Pérez Gómez A. (1993). Modelos contemporáneos de evaluación. En: la evaluación su teoría y su práctica. Caracas: Cooperativa Laboratorio Educativo.

Poggioli, L. (2005). Estrategias de Evaluación. Caracas: Fundación Polar
Rama, G. (1987). Desarrollo y educación en América Latina y el Caribe. Buenos Aires: Kapelusz.

Robbins, S.P.yCoulter,M. (2005).Administración. México: Pearson Prentice Hall.

Tejada, C., Villabona, A. y Ruiz, E. (2013). Deserción y repitencia del programa de ingeniería química de la Universidad de Cartagena. Revista Ciencia e ingeniería al día, 8 (1), 55-66. Recuperado de http://revistas.unicartagena.edu.co/ index.php/ciad/article/view/5

Tinto, V. (1989). Definir la deserción: Una cuestión de perspectiva. Revista de Educación Superior, 71. ANUIES, México.

Valiente, S. (2000). Didáctica de la matemática. El libro de los recursos. Madrid: La Muralla S. A.

Vergel, M., Parra, H \& Martínez, J. (2012) Metodología para elaborar planes de capacitación en instituciones de Educación superior. Revista LOGOS Ciencia \& Tecnología. Recuperado de: http://dx.doi.org/10.22335/rlct.v5i1.388

Vergel-Ortega, M., Gómez-Vergel, C. S., \& Caravalho-Casanova, J. F. (2020). Cuentos sobre las emociones de jóvenes universitarios durante el proceso de aprendizaje del cálculo en ingeniería. Respuestas, 25(1). https://doi. org/10.22463/0122820X.2414 
Vergel Ortega, M., Martínez Lozano J. y and A Ortega Sierra (2019) Validity of microrubri, instrument to measure the development of competences in mathematics IOP Publishing Ltd Journal of Physics: Conference Series, Volume 1160, 1.

Weber, G. (1971). Inner-city children can be taught to read: four successful schools. Washington, DC: Council for Basic Education. 